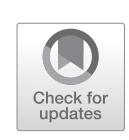

\title{
Robotics in Manufacturing-The Past and the Present
}

\author{
Ming Li, Andrija Milojević, and Heikki Handroos
}

\section{l Robots-From Myth to an Industry Favorite}

Where robots are a household name today the idea and concept of robots dates back to the ancient times. Myths mention artificial people, automatons, and mechanical servants programmed to complete tasks and to serve the Gods. According to ancient Greek myth from 400 BC, Hephaestus, the Greek god of invention and technology, constructed the first automaton (humanoid robot) and called it Talos (see Fig. 1)-this gigantic mechanical warrior in bronze was to guard of the island of Crete $[3,4]$. The automaton had great speed $(250 \mathrm{~km} / \mathrm{h})$, the strength to throw boulders, and an ability to heat-up and crush-burn enemies against his red-hot bronze chest. The mythical Talos was powered by the life-fluid "ichor" that ran in a single artery spanning from the neck to a bolt nailed in the warrior's ankle, the removal of which would cause the life-fluid to flush out and Talos to perish-as the myth would have it that would indeed prove to be the demise of this, perhaps the first, robot.

\footnotetext{
M. Li $(\bowtie) \bullet$ A. Milojević • H. Handroos

Laboratory of Intelligent Machines, LUT-University, Lappeenranta, Finland e-mail: ming.li@lut.fi; andrija.milojevic@lut.fi; heikki.handroos@lut.fi

(C) The Author(s) 2020

M. Collan, K.-E. Michelsen (eds.), Technical, Economic and Societal

Effects of Manufacturing 4.0, https://doi.org/10.1007/978-3-030-46103-4_4
} 


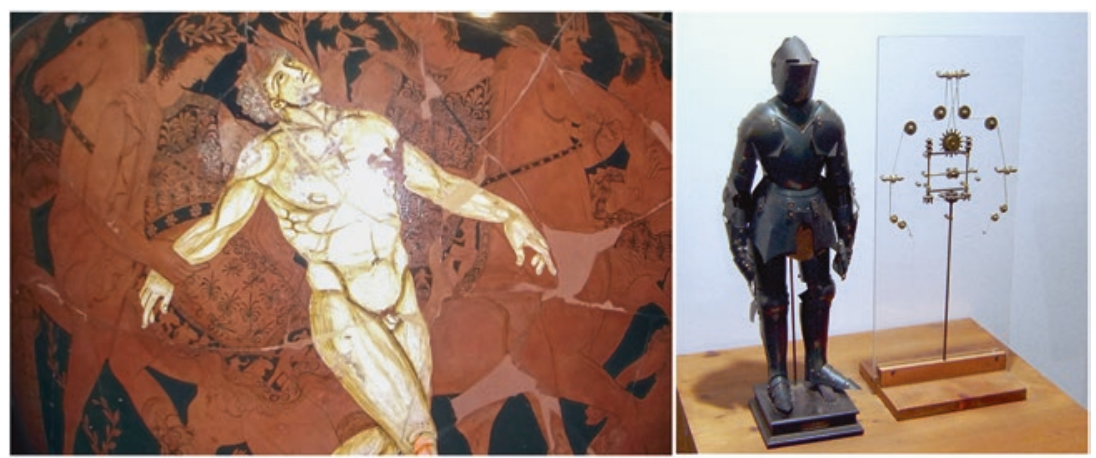

Fig. 1 Detail from an antique vase depicting Talos, (left) [1], Leonardo's automa cavaliere [2]

What the story of Talos and the other ancient myths convey is a story of the ability of humanity to imagine engineered servants or robots already long before our time. One to take the concept further was none other than the great uomo universale Leonardo da Vinci who around the year 1495 designed and constructed a mechanical system called "automa cavaliere" or the mechanical knight. Consisting of cables, pullies, gears, and wheels the knight could be made to perform human-like movement [5]. Based on Leonardo's notes (found only in 1957) a replica of the knight was constructed and the historical accounts on how the original had functioned were corroborated, see Fig. 1.

Until the early twentieth century, mechanical devices with "robotic abilities" were called automatons, or automated mechanical devices-the word "robot" was introduced only in 1920 by the Czech playwright Karel Čapek. In his play “Rossum's Universal Robots” or R.U.R (see Fig. 2), Čapek presents a story of how artificial replicas of humans that have everything except for the soul are constructed to do work that humans don't want to do [7]. Čapek called these artificial men "roboti" based on the Czech word for work. It is said that his original idea was to call them "labori", originally from the latin word "labor" for labor, but that his brother suggested "roboti" and that stuck. At the end of the play the robots rebel against their human masters and finally two robots find love and set off in the sunset to create a new world. Romantic and doomsdayprophetical, the theme has a lot in common with the modern discussion of 


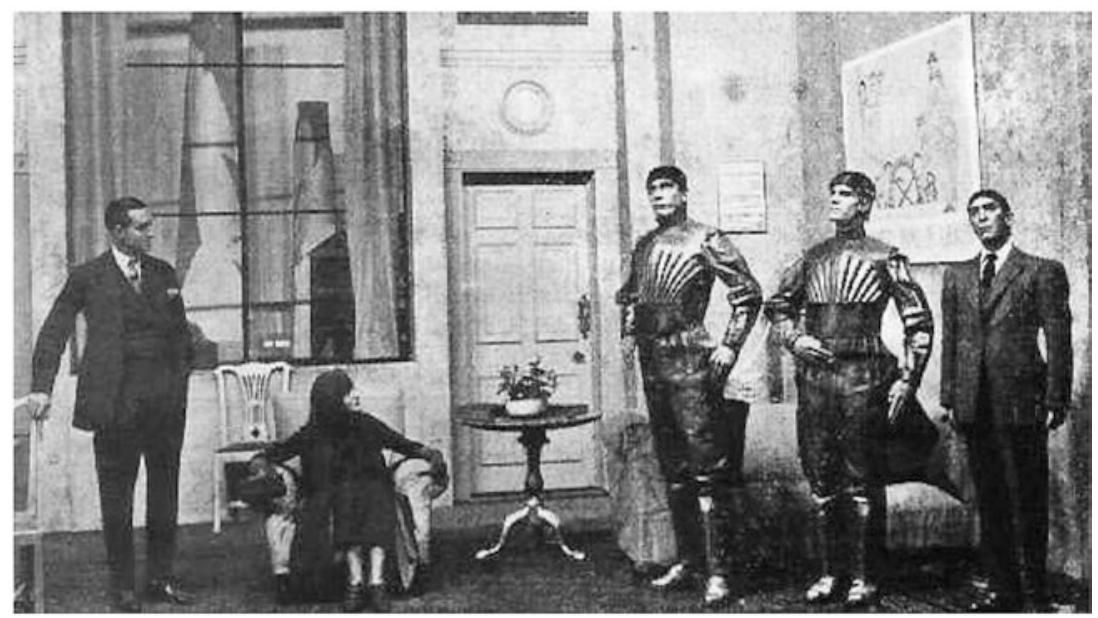

Fig. 2 A scene from the play Rossum's Universal Robots. Three robots are shown on the right [6]

ethics of AI. The word robot was universally adopted and is today used to describe autonomous and automatic machines, especially ones that resemble humans in form.

Although the history of robots is interesting and many visionaries have tried to develop humanoid robots, the most impactful applications of robotics have been in industrial manufacturing, where industrial robots and robotic systems are widely used. The first industrial robots were instances of the robotic arm that depending on the design have several degrees of freedom and that can execute predefined tasks. Industrial robots have dramatically changed the workplace, production processes, and manufacturing, as they have replaced humans in performing many tiresome, dangerous, and repetitive tasks-the change is especially pronounced in areas, where human labor was intensive, harsh, or not even possible. The introduction of robots has generally increased productivity, precision, flexibility, and the quality of production.

The first commercially available and programmable industrial robot was developed by George Devol in 1954 and was called "Unimate" [8]. It was a hydraulics-based manipulator arm that could perform different repetitive tasks. The Unimate had four degrees of freedom, 3 rotations, and 1 
translation and it could lift up to $34 \mathrm{~kg}$, withstand extreme heat, toxic gases, and radio waves. The robot was sold, e.g., to General Motors and implement on their assembly line in 1961. The robot task was to transport cast parts from an assembly line and weld them onto car bodies, which was a dangerous task for human workers. What followed was a massive adoption of robots into the automotive industry that ignited the establishment of many new robotics companies. In Europe, ABB and KUKA Robotics brought industrial robots to the markets in 1973. In 2017 there were 2.5 million robotic units operational worldwide, where the largest number of industrial robots are active in the automotive industry (126,000 units), followed by the electronics industry (121,000 units), and by the metal (45,000 units), plastic and chemical (20,000 units), and food and beverage (10,000 units) industries) [9]. It is estimated by the Industrial Robotics Federation that by 2021 there will be 3.8 million operational industrial robotic units [10].

A shift in the robotics industry has taken place with introduction of Collaborative Robots or "Cobots". A collaborative robot is an industrial robot that is intended to work alongside humans, in occasions, where there is no danger that the robot will hurt the human. The introduction of cobots is something new and for the first-time robots can work alongside humans and "extend" human capabilities, while not aiming to replace them-as cobots are complementary to humans they will most likely drastically shift and change the future of the workplace.

\section{New Trends in Industrial Robotics-Cobots and Advanced Logistics Robots}

With the fast evolution of artificial intelligence technology a new types of industrial robots have emerged to supplement and challenge the on-thegrid and relatively inflexible capabilities of standard industrial robotsintelligent cobots supplement and occupy the flexible manufacturing market. The concept of "cobot" was initially formed from General Motors as early as 1994, and it was literally invented in 1996 by J. Edward Colgate and Michael Peshkin and defined as "an apparatus and method for direct physical interaction between a person and a general purpose manipulator controlled by a computer" in a US patent [11].

Today's production lines are agile with emergence of various customized services and must change timely to the fast-varying market. Standard 
traditional robots are expensive and less versatile in such a scenario and may be out of the reach especially for small and medium-size enterprises that most often require relatively low cost, friendly to use, agile, and fast deployable robots. The intelligent cooperative robots that enter the market can be taught intuitively by operators and can be deployed fast without specific robotic expertise. The range of applications for cobots is wide, from the automotive to the electronics industry, from metal fabrication to packaging and to plastics automation.

In 2008 the UR5 cobot was introduced by Universal Robots in Denmark and in Germany and has rapidly expanded to European and Asian markets. The UR family also has the robots UR3 and UR10 with different payload capacities - all UR robots have an easy programming interfaces and fast set-up, the robots include intuitive and 3D-visualized operations. UR cobots have a high repeatabie $\pm 0.1 \mathrm{~mm}$ position accuracy and they have been applied in a wide range of industrial context. In the presence of coworkers the UR robots can adapt to reduced speeds and even make safety stops with the help of sensors.

In 2011, ABB unveiled a twin-arm cobot, FRIDA (Friendly Robot for Industrial Dual-Arm), originally built for the consumer electronics industry and based on customer desire for a robotic solution for manufacturing environments, where robots and humans must cooperate. A later evolved version of FRIDA was renamed as YuMi (You and Me) and officially introduced to the market by ABB in 2015. The YuMi cobot, with flexible hands, parts feeding systems, and camera-based part location ability is suitable for small parts assembly in a small space, collaborating with human workers. The state-of-the-art control algorithms developed for the cobot can pause its motion within milliseconds, when it encounters an unexpected object-or at even a slight contact with a coworker. The position repeatability of the latest YuMi IRB1400 is $\pm 0.02 \mathrm{~mm}$ with payload of $0.5 \mathrm{Kg}$.

In 2012, "Baxter", the first two-arm collaborative cobot was introduced by rethink robotics, and is usable for a wide range of tasks from packaging and kitting to line loading, machine tending and material handling, and works safely and interactively with coworkers without any caging. A behavior-based user-interface enables the cobot to be programmed intuitively by non-engineers in a matter of minutes. Baxter can also adapt on its own to changes in position and lighting, and to differently shaped objects. Smart sonar systems around the robot head can detect movements within Baxter's proximity, which also enables the robot to learn 
from its environment. The research version of the robot runs Linux and ROS (robot operation system), which are open source and allow further researcher on many aspects of the robot. The later released single-arm cobot, "Sawyer", retains most of the advantages introduced in Baxter, while presenting improved payload performance and accuracy and the ability to execute tasks impractical to automate with traditional industrial robots.

After these early cobots many robot manufacturers have released a number of cobot designs that are more advanced in terms of their ability to monitor their surroundings (typically more cameras and sensors), in the precision of the tasks that they are able to perform (position repeatability), and in their ability to lift higher payloads. Also the application areas of cobots are increasing and their multi-purpose nature is enhanced with the use of smarter and smarter learning technologies that allow deployment and "task training" to be faster in the real-environment.

Logistics robots have for a long time been considered to be outside the scope of industrial robotics, but they are an important part in the complex and dynamic systems of international trade, of which industry is a part of. Successful applications of logistics robots, such as the autonomous pick and place robots by, for example, Amazon and DHL in their warehouses have greatly increased efficiency in order picking and other warehouse tasks. Even in very large warehouses and their dynamic environments the workflows can be set up and modified quickly with the help of intelligent autonomous logistic robots and robotic data cloud systems. International eCommerce and logistics companies that own and operate warehouses have diversified into robotics by acquiring some logistics robotics companies. It is visible that robotics have become a source of competitive advantage in logistics and the optimization of the use of logistics robots is a way to further enhance the productivity of robotic systems these include, among others, optimization and coordinated autonomy of logistics robots. The management of the robot-fleet is tied with the overall management of a warehouse and tied with demand forecasting based on machine learning algorithms-advanced multi-modal systems allow for real-world order picking to start taking place before a customer has even finished making her selections in a virtual eStore.

The next generation logistics robots are envisaged to be vision guided AGVs (Automatic Guided Vehicles) and "perception" and the ability to grasp objects are perhaps the main areas where development is neededartificial intelligence combined with ranging sensors and cameras will 
enable robots to identify, classify, and grasp objects from the best direction and in the best position. Unlike the classic AGV systems that rely on the physical path guidance in the form of embedded magnets, wires, painted lines, magnetic tapes, reflectors, or other path-defining means, the SLAM (simultaneous localization and mapping) algorithms have are used by many modern robots - these robots can autonomously create a map of an unknown environment and maintaining knowledge of their own location within the created map. Typically the mapping is achieved through scanning with a $2 \mathrm{D}$ or a $3 \mathrm{D}$ Lidar, often supported with a $3 \mathrm{D}$ stereo depth camera with advanced sensing-combining the odometer and some advanced filtering techniques allows the robots to estimate quite precisely their position on the map, while being able to avoid unknown (even moving) obstacles on their path. Logistics robots with this kind of capability are able to operate autonomously also in locations "they have never seen before"-unsurprisingly similar technologies are commonly used in robotic vacuum cleaners in household use. It can be seen that there is a merger of robotics with artificial intelligence going on that will result in more precisely and autonomously functioning robots that are multiusable. In fact, sharing and copying the characteristics that have been found to be successful in the context of logistics robots in the large e-shopping warehouses, large manufacturing factories are also adopting logistics robots to support manufacturing operations.

Where many cobots are fully autonomous, also remotely operated systems can be considered cobot systems. There are still many places where humans are needed to operate machines and systems that require more cognitive skills than the automation of today can provide. In the future, the emerging wireless communication technologies will be able to provide a sufficiently small latency to carry out work tasks that require multisensory feedback to the remote operator-making such fast data transfer available globally may significantly change the labor markets at least in some niche areas, as remote operation of devices and machines by skilled operators can be done from anywhere in the world. Fast 3D cameratechnology, virtual helmets, and haptic interfaces may someday provide the remote operator with a close to a fully realistic feeling of presence in the actual machine performing the task.

All in all it can be observed that industrial robotics is a highly interdisciplinary area that covers many fields from mechanical-, electronics-, and dynamics design, to construction of actuators and servo driving technology, to signal processing and control, and to AI and software development. 


\section{Supporting Technologies Behind Modern Robotics}

Machine vision has now become an important integral part of perception ability of an intelligent industrial robot, which enables a robot to recognize objects it is handling and to position the end-effector through inhand vision, and to perceive and to construct a cooperative environment for human coworkers. Convolutional neural networks play an important role in how machine vision is put into practice today-they are used already quite commonly in image classification and in object detection and tracking. These skills are interesting, for example, when industrial robots are used in product quality control.

As one of the targets for industrial robots is to function in a "partner" role with human coworkers, while being autonomous, reinforcement learning is an important tool in enabling robots to learn "behavior" through interaction [12]. A variety of complex problems, where no obvious programmable pattern and behavior appears to be discernible due to the environmental dynamics or uncertainty, can be solved with reinforcement learning - such problems include force control [10] used, for example, in robotic hands and as technology to optimize robots grasping things and for motion planning [13-15].

Historically artificial neural networks have been applied in robotics for a long time, mostly via their applications to various control problems. One direction, where the study of artificial neural networks has gone in the context of robotics is reservoir computing [15].

Evolutionary algorithms have become an important search and optimization technique within the last two decades, since robotics contains high dimensional parameters that lead to identification and optimization problems, evolutionary algorithms have been actively applied to robotics, covering areas such as kinematic and dynamic parameter identification, controller parameter optimization, trajectory generation, and motion planning. Evolutionary algorithms are just one type of optimization tool however, for one reason or another, most likely the malleability of these methods fit many types of problems, and they have been often used in the context of robotics. Typically the optimization is done off-line due to the computational cost, which means that evolution of a robot in terms of optimization and reoptimization is step-wise-in the future real-time re-optimization will become possible and the advanced sensors that provide robots with high quality real-time situational awareness can be used to generate optimization problems that are solved instantly and the robot acts accordingly. 


\section{Conclusions}

Although the origins of the industrial robot were in the field of automobile assembly, its application has expanded into virtually all fields of manufacturing and process industries. With the advances made in artificial intelligence that translate to better ability to control the physical functionality of robots and sensor technology that allow robots to acquire sensory skills that match those of humans it can be forecasted that intelligent robots may become ubiquitous is manufacturing, and elsewhere in the society. Robots that have learning capabilities may not only boost the productivity over a wide variety of application areas in manufacturing, but may also provide a basis for using robots in tasks where they have not previously been used. A learning robot that learns in a place where it originally was not designed to learn can be said to be a step closer to an allpurpose robot and becomes a "method of invention". Importantly, modern industrial robots are not any more limited by their ability working on a single task alone, but possess abilities that allow them to be used in connection with various different task the level of difficulty of which is increasing. The enhanced learning abilities present in modern intelligent robots are an important step in terms of general applicability of robotslooking at this situation from the point of view of economics, a robot with the capability to handle a large number of tasks that can easily be taught to handle "any compatible task" has the advantage and potential of becoming a standard tool for various tasks. In this vein, it is not wrong to expect that manufacturing will continue to be disrupted by robotics in the future. On the other hand, it should be remembered that technological developments in robotics and automated manufacturing systems don't take place in a vacuum. It is too simple to argue that advancements in technology shape social structures and social potential. The critical history of robotic and automated manufacturing systems demonstrates an opposite reality. Technological potential embedded in industrial robots and manufacturing systems has often been limited by social constrains. Efforts to replace human labor and to create industrial processes that exist without people, have been hampered by political, economic, and ideological tensions. It has been important to soften the disruption and to present that robots and automated manufacturing systems are "collaborators". Machines will take over human work and perform difficult and dangerous tasks, but the ultimate command will be in the hands of skilled workers and managers. It is difficult or perhaps even impossible to predict, for how long human labor is needed. Technology optimists suggest that the power has already shifted 
and the advanced systems are taking over also managerial tasks. The opposite argument emphasizes typically historical experience. What can be said is that social structures within manufacturing industries are strong and resilient, while the human mind is able to cope with even rapid technological advances.

\section{REFERENCES}

1. Photo: Forzaruvo94 / CC BY-SA (https://creativecommons.org/licenses/ by-sa/3.0).

2. Photo by Erik Möller. Leonardo da Vinci. Mensch—Erfinder-Genie exhibit, Berlin 2005./Public domain.

3. A. Mayor, "Gods and Robots: Myths, Machines, and Ancient Dreams of Technology", Princeton University Press, 2018.

4. R. Apollonius, "The Argonautica", CreateSpace Independent Publishing Platform, 2017.

5. R. Mark, “Leonardo's Lost Robots”, Springer Berlin Heidelberg, 2016.

6. Photo: Unknown, uploaded to ar.wiki by ar: Classic 971 on 13 July 2006, to en.wiki by en:User:Rocketmagnet on 4 December 2007, to commons by User:Sreejithk2000 on 2 June 2010/Public domain.

7. K. Capek, “R.U.R.: (Rossum's Universal Robots)”, Players Press, 2002.

8. A. Gasparetto, and L. Scalera, From the Unimate to the Delta Robot: The Early Decades of Industrial Robotics" in: Proceedings of the 2018 HMM IFToMM Symposium on History of Machines and Mechanisms. https://doi. org/10.1007/978-3-030-03538-9_23, 2019.

9. Executive Summary World Robotics 2019 Industrial Robots, International Federation of Robotics (IFR), 2019.

10. R. Meyes et al., "Motion Planning for Industrial Robots using Reinforcement Learning," Procedia CIRP, vol. 63, pp. 107-112, 2017.

11. "Cobots" US Patent 5,952,796, https://patents.google.com/patent/ US5952796.

12. F. Stulp, E. Theodorou, J. Buchli, and S. Schaal, "Learning to grasp under uncertainty," Proc.-IEEE Int. Conf. Robot. Autom., pp. 5703-5708, 2011.

13. M. Vecerik, O. Sushkov, D. Barker, T. Rothorl, T. Hester, and J. Scholz, "A practical approach to insertion with variable socket position using deep reinforcement learning," Proc.-IEEE Int. Conf. Robot. Autom., vol. 2019-May, pp. 754-760, 2019.

14. Y. Litvak et al. "Learning a High-Precision Robotic Assembly Task Using Pose Estimation from Simulated Depth Images." ArXiv abs/1809.10699 (2018):n.pag.

15. A.S. Polydoros, L. Nalpantidis and V. Kruger, "Advantages and Limitations of Reservoir Computing on Model Learning for Robot Control", Paper presented at IROS Workshop on Machine Learning in Planning and Control of Robot Motion, Hamburg, Germany. 
Open Access This chapter is licensed under the terms of the Creative Commons Attribution 4.0 International License (http://creativecommons.org/licenses/ by $/ 4.0 /$ ), which permits use, sharing, adaptation, distribution and reproduction in any medium or format, as long as you give appropriate credit to the original author(s) and the source, provide a link to the Creative Commons licence and indicate if changes were made.

The images or other third party material in this chapter are included in the chapter's Creative Commons licence, unless indicated otherwise in a credit line to the material. If material is not included in the chapter's Creative Commons licence and your intended use is not permitted by statutory regulation or exceeds the permitted use, you will need to obtain permission directly from the copyright holder.

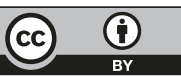

